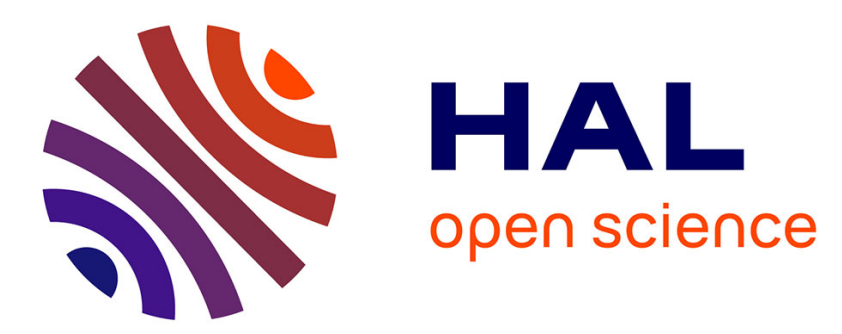

\title{
Eyesafe coherent detection wind lidar based on a beam-combined pulsed laser source
}

\author{
L. Lombard, M. Valla, C. Planchat, D. Goular, B. Augere, Pascal Bourdon, G.
}

Canat

\section{- To cite this version:}

L. Lombard, M. Valla, C. Planchat, D. Goular, B. Augere, et al.. Eyesafe coherent detection wind lidar based on a beam-combined pulsed laser source. Optics Letters, 2015, 40 (6), p. 1030-1033. 10.1364/OL.40.001030 . hal-01228416

\section{HAL Id: hal-01228416 https://hal.science/hal-01228416}

Submitted on 14 Feb 2022

HAL is a multi-disciplinary open access archive for the deposit and dissemination of scientific research documents, whether they are published or not. The documents may come from teaching and research institutions in France or abroad, or from public or private research centers.
L'archive ouverte pluridisciplinaire HAL, est destinée au dépôt et à la diffusion de documents scientifiques de niveau recherche, publiés ou non, émanant des établissements d'enseignement et de recherche français ou étrangers, des laboratoires publics ou privés. 


\title{
Eyesafe coherent detection wind lidar based on a beam-combined pulsed laser source
}

\author{
L. Lombard,* M. Valla, C. Planchat, D. Goular, B. Augère, P. Bourdon, and G. Canat \\ Onera, The French Aerospace Lab, Palaiseau, France \\ ${ }^{*}$ Corresponding author: Laurent.Lombard@onera.fr
}

\begin{abstract}
We report on a coherent wind lidar built with two coherently-beam-combined fiber amplifiers. The lidar perfor-mances of the combinedamplifier and the single-amplifier are compared using two criterions: carrier-to-noise ratio and wind speed noise floor. In both cases, lidar performances are not degraded with a combined source and are close to the theoretical optimum. Combined sources are well suited to improve coherent wind lidar accuracy, range, and integration time.
\end{abstract}

Coherent wind lidars are increasingly used for wind measurement and turbulence assessment with applications in wind farm project optimization or aircraft security during take-off and landing. Laser pulses are emitted through the atmosphere, and wind speed is measured using Doppler-induced frequency shift on the backscattered laser light [1]. Laser sources with excellent spatial beam quality, narrow linewidth, and typical pulse duration ranging from $\sim 100 \mathrm{~ns}$ to $1 \mu \mathrm{s}$ are required. Pulsed master oscillator power fiber amplifiers (MOPFAs) at $1.5 \mu \mathrm{m}$ are well adapted, versatile sources but with peak power limited in typical fibers to a few $100 \mathrm{~s} \mathrm{~W}$ by nonlinear effects, especially stimulated Brillouin scattering (SBS).

Coherent beam combination (CBC) allows improving the output power of MOPFA sources by adding the outputs of single amplifiers. To achieve this, two or more amplifiers seeded by the same pulsed oscillator are coherently combined into a single-mode beam. To achieve high CBC efficiency, the phase differences are compensated using a controller. We have recently demonstrated the $\mathrm{CBC}$ of two amplifiers in a $100 \mathrm{~ns}$ pulse regime limited to 95 and $123 \mathrm{~W}$, resulting in peak power of $208 \mathrm{~W}$ [2]. Beam quality and spectral linewidth were maintained. The performance of this source is promising for coherent lidar, but the compatibility with $\mathrm{CBC}$ controller requires verification because of additional noise introduced by the frequency tagging.

In this communication, we report on the use of a CBCbased source to improve coherent detection wind lidar. In particular, we demonstrate that the pulse operation CBC phase controller does not impair the lidar performances.

The principle of wind lidar is shown in Fig. 1. A CW master oscillator is externally modulated into pulses with $240 \mathrm{~ns}$ pulse duration and frequency shifted by $100 \mathrm{MHz}$ using an acousto-optical modulator (AOM). Pulses are amplified in one or more successive fiber amplifiers to reach about $50 \mathrm{~W}$ peak power and sent out into the atmosphere. Light is backscattered by aerosols along its path in the atmosphere. A circulator made of a polarizing beam splitter and a quarter waveplate enables coupling of backscattered light into a single mode fiber, thanks to the polarization-preserving property of the aerosols.

The collected backscattered light is then mixed with the local oscillator to produce interferences on a heterodyne photo-detector. The aerosol velocity can be directly measured from the coherent mixing beat frequency. Indeed, the heterodyne current produced by the detector can be written as

$i_{\text {het }}=\sqrt{2 \cdot K \cdot P_{S} \cdot P_{\mathrm{OL}}} \cdot \cos \left[2 \pi \cdot\left[\left(f_{e}-f_{\mathrm{OL}}\right) \pm f_{D}\right] \cdot t+\varphi\right]$,

where $f_{D}=2 \cdot \frac{V_{r}}{\lambda}$ is the Doppler frequency shift; $P_{S}$ and $P_{\mathrm{OL}}$ are the backscattered and local oscillator laser powers; $f_{e}$ and $f_{\mathrm{OL}}$ are the emitted and local oscillator frequencies; and $K$ is a constant and $\varphi$ is a phase. $V_{r}$ is the wind speed projection on the laser path. The analysis of the carrier frequency of $i_{\text {het }}$ leads to the wind speed $V_{r}$.

A useful coherent lidar signal quality criterion is the carrier-to-noise ratio (CNR) that can be written as [3]

$$
\mathrm{CNR}=\frac{\left\langle i_{\text {het }}^{2}\right\rangle}{\left\langle i_{\text {noise }}^{2}\right\rangle} \propto \frac{P_{L}}{D^{2}},
$$

where $\left\langle i_{\text {het }}^{2}\right\rangle$ is the average power of the heterodyne current, $\left\langle i_{\text {noise }}^{2}\right\rangle$ is the average power of the noise current on

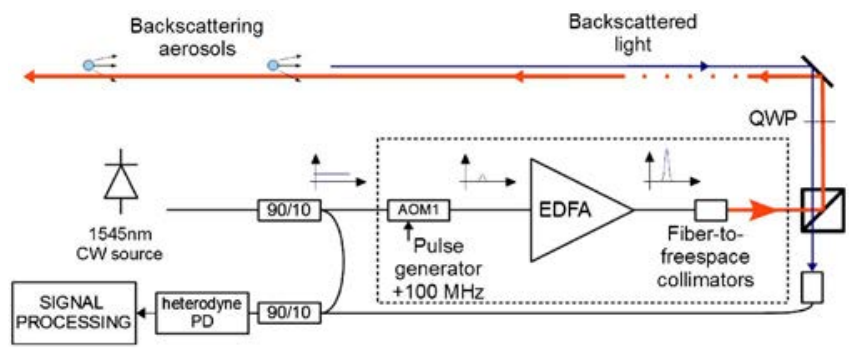

Fig. 1. Principle of a wind lidar. AOM, acousto-optic modulator; EDFA, erbium doped fiber amplifier; QWP, quarter waveplate; PD, photodiode. The quarter wave allows coupling of back-scattered light to collection fiber optics. The dashed rectangle delimits the pulse amplifier to be replaced by a combined amplifier. 
the detector, $P_{L}$ is the source peak power, and $D$ is the observation distance. The maximum lidar range is obtained when the CNR reaches the CNR threshold required by signal processing. Note that for the sake of simplicity, CNR has been admitted to be inversely proportional to the square of distance, which is an assumption only valid for long-range collimated lidars. A more accurate expression of CNR can be obtained in ref. [4].

In the usual case of the noise being limited by detection noise, the accuracy of the measured wind speed is theoretically proportional to

$$
\sigma_{V} \propto \frac{1}{f(\mathrm{CNR}) \sqrt{N}},
$$

where $N$ is the number of averaged pulses and $f$ is an increasing function depending on lidar configuration. More information on computation of $f$ function can be found in Ref. [5]. By increasing the CNR, we can thus improve the measurement range, reduce the integration time, or improve the speed accuracy.

According to Eq. (2), a peak power improvement by a factor $M$ using a beam combination of $M$ sources should then lead to a CNR improvement of a factor $M$ and a lidar range improvement of a factor $\sqrt{M}$. To demonstrate this, we first check using $M=2$ sources that the CBC does not degrade lidar performances and then show that expected CNR improvement is reached.

In Fig. 1, we have replaced the fiber amplifier EDFA and its collimator, identified by the dashed rectangle, by a combined amplifier shown in Fig. 2 . The input signal is first split into two beams, amplified separately in two EDFAs, combined on output $\mathrm{O}_{1}$, and sent into the atmosphere. A coherent pulse combination at output $\mathrm{O}_{1}$ is maintained through the minimization of output $\mathrm{O}_{2}$ by the LOCSET phase control system modified for pulse operation [ $[\underline{6}, 2]$. The first modification is the use of a signal leak between the pulses for phase measurement. The second modification is the presence of a pulse suppressor before LOCSET photodiode to avoid the photodiode saturation by the high pulse power. This technique requires that the phase difference not change significantly during the pulse. This was demonstrated in ref. [2]. For more details on the combined source in pulsed operation, see ref. [2]. Figure $\underline{3}$ shows the timing diagram for combined amplifier in arbitrary scales. In this particular

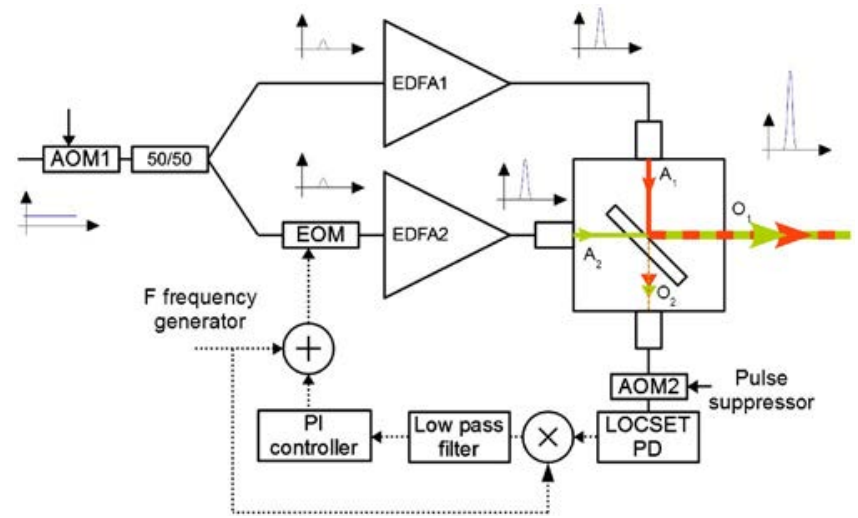

Fig. 2. Coherently combined pulsed amplifier setup. combined source, the leak is removed during the first microseconds (AOM1 off-time) after each pulse to avoid perturbation by back-reflected signal leak while the heterodyne current is measured. Indeed, parasitic reflexion of a signal leak on output optics is non-negligible compared to atmospheric signal and can prohibit lidar measurement. Basically, heterodyne measurement operates during AOM1 off-time and LOCSET operates during AOM1 leak-time.

To demonstrate the compatibility of pulsed CBC with wind lidar requirements, we compare the performances of a wind lidar using a $30 \mathrm{~W}$ peak power source, either made of a single-amplifier (mode A, see Table 1) or of two combined amplifiers (mode B). The output peak power was limited to $33 \mathrm{~W}$ in mode $\mathrm{B}$ for the sake of comparison. The amplifiers are limited to $\sim 50 \mathrm{~W}$ by SBS. An additional mode (C) uses both amplifiers at full power (96 W peak power). The pulse's duration is $240 \mathrm{~ns}$ with $10 \mathrm{kHz}$ pulse repetition frequency.

The lidar is alternatively operated for $10 \mathrm{~min}$ in each mode A, B, and C with fast ( $6 \mathrm{~s})$ switching time, to probe the atmosphere in the most similar state for all modes. This cycle has been repeated over a few days. The laser beam of the lidar is aimed at the atmosphere with an elevation angle of $45^{\circ}$. The telescope diameter is $5 \mathrm{~cm}$, and the beam is collimated for long-range operation.

The backscattered signal is acquired for each pulse, and its frequency shift is analyzed for seven distances, ranging from 100 to $400 \mathrm{~m}$, using the following procedure. A power spectral density (PSD) is computed for each distance and laser shot. Then PSDs are averaged for 1,000 shots, corresponding to $0.1 \mathrm{~s}$ acquisition time, resulting in seven PSDs, ready to be analyzed for Doppler shift. PSD analysis includes an estimation of the CNR, defined as the ratio of the power contained in the signal to the power contained in the noise over the full detector bandwidth, and an estimation of the wind speed through a

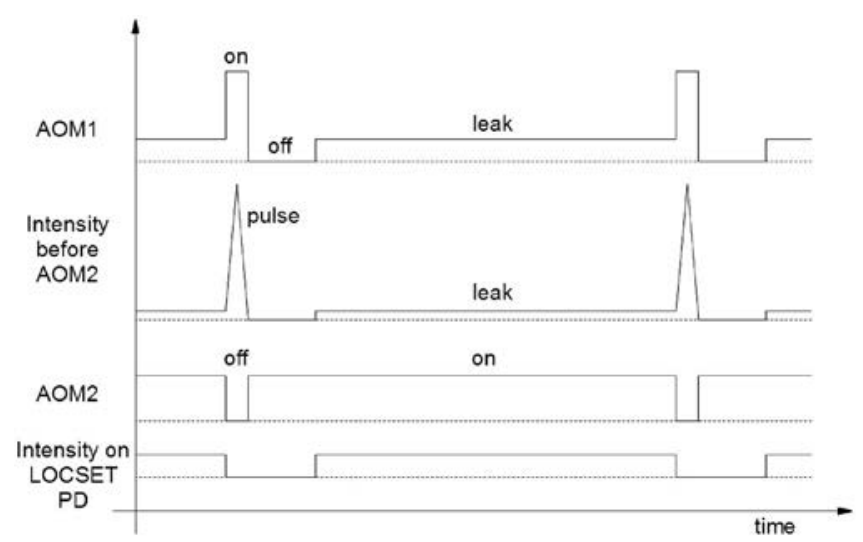

Fig. 3. Timing diagram of AOMs 1 and 2 and intensities in combined pulse amplifier setup of Fig. 2 .

Table 1. Combined Source Configurations

\begin{tabular}{cccc}
\hline & Amplifier 1 (W) & Power Amplifier 2 (W) & Output $\mathrm{O}_{1}(\mathrm{~W})$ \\
\hline $\mathrm{A}$ & 49 & - & 29 \\
$\mathrm{~B}$ & 21 & 13 & 33 \\
$\mathrm{C}$ & 49 & 51 & 96 \\
\hline
\end{tabular}



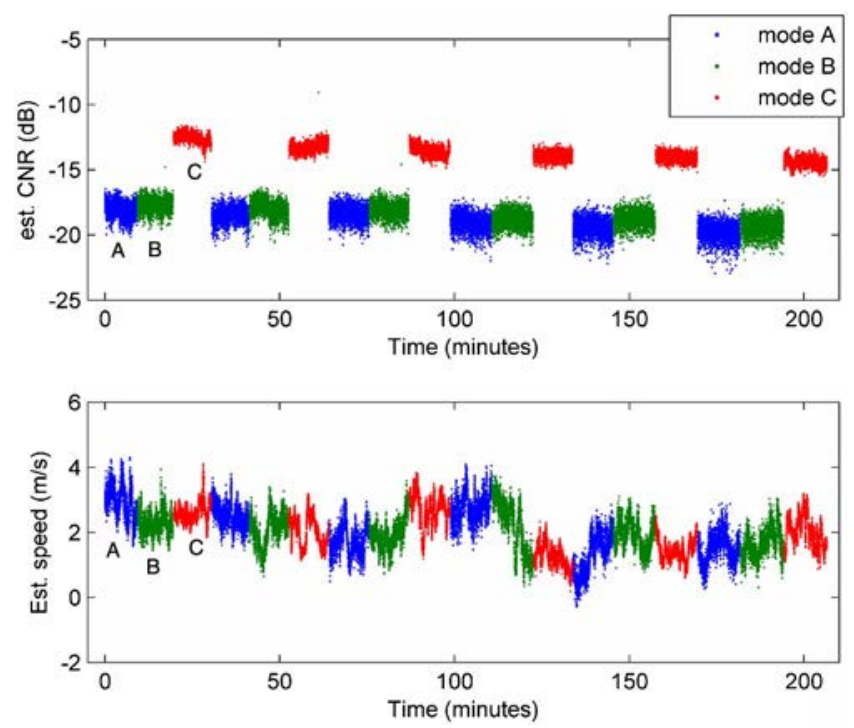

Fig. 4. Lidar performance comparison at $150 \mathrm{~m}-\mathrm{CNR}$ and estimated speed. Mode A, B and C are alternatively used on a period of $\sim 10 \mathrm{~min}$.

maximum likelihood estimator (MLE) [7]. The CNR and wind speed are being estimated jointly with the signal spectral width.

Figure 4 shows the time series of estimated CNR (top) and of estimated wind speed (bottom) for a distance of $150 \mathrm{~m}$ from the emission pupil. Sampling time is $300 \mathrm{~ms}$. Acquisition time is $10 \mathrm{~min}$ in each mode. Figure $\underline{5}$ (left) shows that the time evolution of CNR is all modes. The slow CNR decrease can be related to aerosol concentration drop. Figure 5 (right) shows that the CNR difference is stable in time. The $0.55 \mathrm{~dB}$ increase from mode $\mathrm{A}$ to mode $\mathrm{B}$ is related to the $0.57 \mathrm{~dB}$ power increase at output $\mathrm{O}_{1}$, and the $5.5 \mathrm{~dB}$ increase from mode $\mathrm{A}$ to mode $\mathrm{C}$ is related to the $5.6 \mathrm{~dB}$ power increase. The estimated wind speed varies in time with continuity between modes. Those CNR measurements show no degradation because of the CBC system.

Figure $\underline{6}$ is a detail of the first cycle of Fig. $\underline{4}$. We can observe that the wind speed obtained at a larger CNR shows less dispersion. One way to measure the accuracy of a wind sensor is to measure the noise floor of the PSD of the estimated wind speed data. Estimated data are the sum of the wind data and the estimator error. Estimator error is a white noise, as there is no correlation between
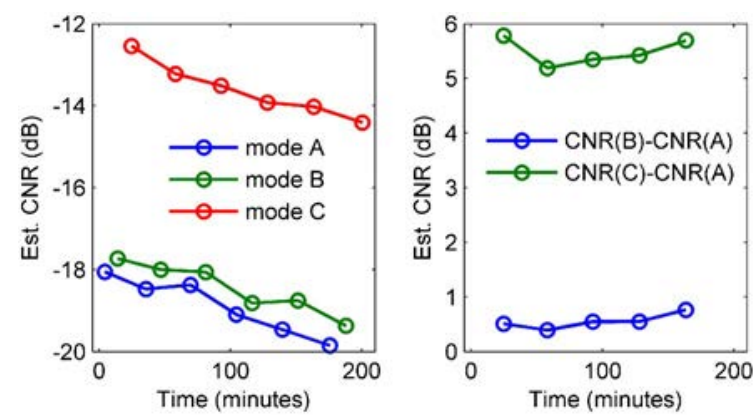

Fig. 5. (left) CNR averaged over 10 min in modes A, B, and C; (right) average $\mathrm{CNR}$ difference: mode $\mathrm{B} \mathrm{CNR}$-mode $\mathrm{A}$ CNR and mode C CNR-mode A CNR.

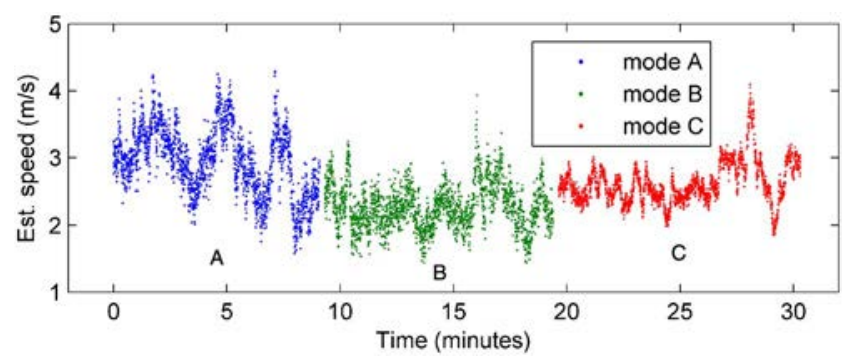

Fig. 6. Detail of the first cycle of Fig. $\underline{4}$.

errors at two different times. Wind data fluctuation is the consequence of wind turbulence, and follows a Kolmogorov law: its PSD is proportional to $f^{-\frac{5}{3}}$ [8] .

Figure 7 shows the PSD of the three 10 min wind data sets shown in Fig. 5. Each set is split in $10 \sim 1$-min data subsets. Then, the PSDs of the 10 subsets are averaged to smooth out high frequency noise. On each PSD log-log graph we can visualize the $-\frac{5}{3}$ slope of the wind turbulence, and the flat level of the estimator noise [9]. The vertical axis value is expressed in terms of equivalent wind speed variance (the PSD value has been integrated over the band). The experimental wind speed accuracy, reported in Table 2, is similar between single amplifier (mode A) and beam combining (mode B), and is improved in mode C. Again, this result proves that beam combining does not degrade the wind speed accuracy and complies with coherent Doppler lidar requirements. Beam combining at full power (mode C) yields a better wind speed accuracy than with a single amplifier (mode A). The theoretical performance of the wind speed estimator, given by Cramér-Rao bound [5,7], has been computed from CNR estimated values and is illustrated by the green line in Fig. 7 and reported in Table 2 . Wind speed performance obtained by the lidar is very close to its theoretical optimum in all modes.

In summary, the CBC is a scalable technique for improving output power of single-frequency pulse sources used in lidar and other fields. We have achieved the efficient CBC of two 240-ns, single-mode, single-frequency pulses from SBS-limited amplifiers. This coherently combined pulse source has been implemented in a wind lidar with associated signal processing. The performance of the lidar has been evaluated through two different techniques: CNR estimation in the measured spectra and noise floor evaluation of the measured wind speed.

In both cases, no performance degradation has been measured when using CBC. When both amplifiers are run at full power, the expected lidar performance improvement is reached. The lidar performances are very close to its optimum.

Those results show the compatibility of coherent beam combining with coherent wind lidar. $\mathrm{CBC}$ opens the way for improvement of lidar reliability and measurement range.

The number of sources that can be combined is currently limited by the optical configuration chosen for combination to only a few. However, optical configurations compatible with LOCSET can be used to improve this number. In a future work, the $50 \mathrm{~W}$ limited singlemode-fiber based sources will be replaced by LMA-fiber based sources. 

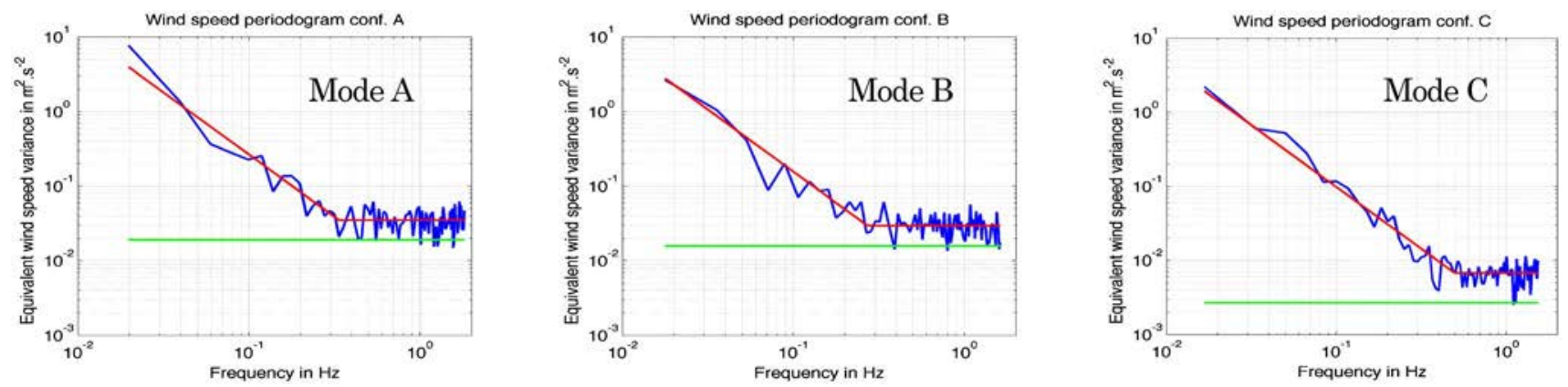

Fig. 7. Lidar performance comparison at $150 \mathrm{~m}$. Instrument noise floor comparison: blue, PSD of estimated wind speed from Fig. 6; red (low freq.), $\alpha f^{-\frac{5}{3}}$ Kolmogorov law ( $\alpha$ fitted to data); red (high freq.), lidar noise floor fitted to data; green, lidar theoretical noise floor from estimated CNR.

Table 2. Lidar Performance Comparison

\begin{tabular}{cccc}
\hline & $\begin{array}{c}\text { Wind Speed } \\
\text { Accuracy }(\mathrm{m} / \mathrm{s})\end{array}$ & $\begin{array}{c}\text { Cramér-Rao } \\
\text { Bound }(\mathrm{m} / \mathrm{s})\end{array}$ & $\begin{array}{c}\text { Accuracy Gain w.r.t. } \\
\text { Mode A (dB) }\end{array}$ \\
\hline A & $0.21 \pm 0.02$ & $0.15 \pm 0.02$ & \\
B & $0.19 \pm 0.02$ & $0.14 \pm 0.02$ & 0.37 \\
C & $0.08 \pm 0.006$ & $0.06 \pm 0.006$ & 4.1 \\
\hline
\end{tabular}

\section{References}

1. S. W. Henderson, P. Gatt, D. Rees, and R. M. Huffaker, in Laser Remote Sensing, T. Fujii and T. Fukuchi, eds. (CRC Press, Taylor and Francis Group, 2005) pp. 469-722.
2. L. Lombard, A. Azarian, K. Cadoret, P. Bourdon, D. Goular, G. Canat, V. Jolivet, Y. Jaouën, and O. Vasseur, Opt. Lett. 36, 523 (2011).

3. C. M. Sonnenschein and F. A. Horrigan, Appl. Opt. 10, 1600 (1971).

4. R. Frehlich and M. J. Kavaya, Appl. Opt. 30, 5325 (1991).

5. B. J. Rye, Appl. Opt. 39, 6086 (2000).

6. T. M. Shay, V. Benham, J. T. Baker, B. Ward, A. D. Sanchez, M. A. Culpepper, D. Pilkington, J. Spring, D. J. Nelson, and C. A. Lu, Opt. Express 14, 12015 (2006).

7. R. Frehlich, J. Atmos. Oceanic Technol. 30, 230 (2013).

8. A. S. Monin and A. M. Yaglom, Statistical Fluid Mechanics: Mechanics of Turbulence (MIT, 1975) Vol. 2, p. 874.

9. R. Frehlich, S. M. Hannon, and S. W. Henderson, Bound.Lay. Meteorol. 86, 233 (1998). 